\title{
Influência da hipovitaminose D na ocorrência das fraturas*
}

\section{Influence of Hypovitaminosis D on the Occcurrence of Fractures}

\author{
Cleandro Augusto Ribeiro do Valle ${ }^{1}$ Luiz Alfredo Furtado Pedri ${ }^{10}$ Guilherme Bigonha Pires ${ }^{10}$ \\ Mariella Soares Blanco ${ }^{20}$ Bruna Soares Paula ${ }^{20}$ Carlos Delano Mundim Araújo $1,3(0)$ \\ ${ }^{1}$ Departamento de Ortopedia e Traumatologia, Hospital das Clínicas \\ Samuel Libânio, Universidade do Vale do Sapucaí (Univás), Pouso \\ Alegre, Minas Gerais, MG, Brasil \\ 2 Faculdade de Ciências Médicas Dr. José Garcia Coutinho, Universidade do \\ Vale do Sapucaí (Univás), Pouso Alegre, Minas Gerais, MG, Brasil \\ ${ }^{3}$ Centro Universitário Atenas (UniAtenas), Paracatu, Minas Gerais, \\ MG, Brasil \\ Endereço para correspondência Carlos Delano Mundim Araújo, M.D, \\ Hospital das Clínicas Samuel Libânio, Rua Comendador José Garcia \\ 777, Centro, Pouso Alegre, Minas Gerais, MG, 37550-000, Brasil (e- \\ mail: drcdelano@hotmail.com). \\ Rev Bras Ortop 2021;56(6):784-789.
}

\begin{abstract}
Resumo
Palavras-chave

- vitamina D

- deficiência de vitamina D

- osteoporose

- fraturas do quadril

- consolidação da fratura

Objetivo Dosar a vitamina $\mathrm{D}$ nos pacientes internados por fratura e avaliar sua relação com as fraturas.

Métodos Realizou-se estudo primário, analítico, transversal, não intervencional, observacional, controlado, em seres humanos. Fez-se a dosagem sérica de: vitamina D, paratormônio(PTH), cálcio, fósforo, magnésio e albumina em 49 pacientes que foram internados consecutivamente para cirurgia devido a fratura(s) (grupo de estudo), e em 50 pacientes, dos diversos ambulatórios, sem fraturas, e que realizaram exames de rotina (grupo de controle).

Resultados A média de 25-hidroxivitamina $\mathrm{D}(25[\mathrm{OH}] \mathrm{D})$ nos pacientes com fraturas foi de $23,78 \pm 8,01 \mathrm{ng} / \mathrm{mL}$ (61,22\% dos pacientes com fraturas apresentaram hipovitaminose $\mathrm{D}$ ). A média de $25(\mathrm{OH}) \mathrm{D}$ do grupo de controle foi de $37,52 \pm 9,21 \mathrm{ng} / \mathrm{mL}$ ( $10 \%$ dos pacientes apresentaram hipovitaminose D). A maioria dos casos de hipovitaminose (96\%) não cursou com hiperparatireoidismo secundário (média do PTH $=41,80 \pm 22,75 \mathrm{pg} / \mathrm{mL}$ ).

Conclusão A hipovitaminose $D$ foi encontrada em um percentual expressivo $(61,22 \%)$ dos pacientes que sofreram fraturas. Mais estudos sobre o assunto são necessários para se compreender melhor a influência da hipovitaminose D na ocorrência das fraturas e os benefícios da suplementação com vitamina $\mathrm{D}$ nesses pacientes.
\end{abstract}

\footnotetext{
Trabalho desenvolvido no Departamento de Ortopedia e Traumatologia do Hospital das Clínicas Samuel Libânio, Pouso Alegre, Minas Gerais, MG, Brasil.
}

recebido

26 de Outubro de 2019

aceito

01 de Dezembro de 2020

Publicado on-line

Novembro 11, 2021
DOI https://doi.org/ $10.1055 / \mathrm{s}-0041-1729585$. ISSN 0102-3616.

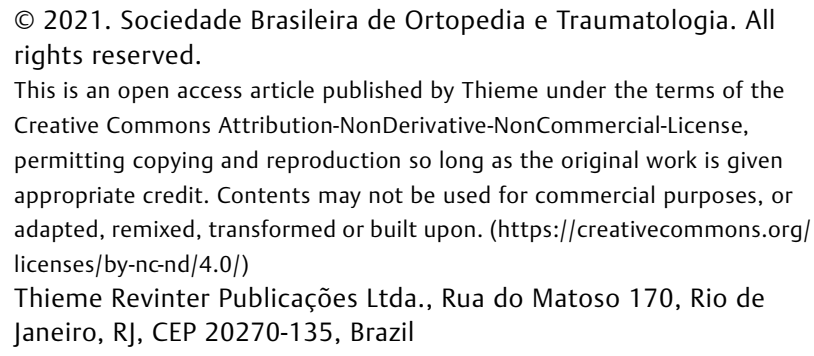

(c) 2021. Sociedade Brasileira de Ortopedia e Traumatologia. All rights reserved.

This is an open access article published by Thieme under the terms of the Creative Commons Attribution-NonDerivative-NonCommercial-License, permitting copying and reproduction so long as the original work is given appropriate credit. Contents may not be used for commercial purposes, or adapted, remixed, transformed or built upon. (https://creativecommons.org/ licenses/by-nc-nd/4.0/) Thieme Revinter Publicações Ltda., Rua do Matoso 170, Rio de Janeiro, RJ, CEP 20270-135, Brazil 
Abstract

\section{Keywords}

- vitamin D

- vitamin de deficiency

- osteoporosis

- hip fractures

- fracture healing
Objective To measure the levels of vitamin $D$ in patients hospitalized for fractures and to evaluate its relationship with fractures.

Methods A primary, analytical, cross-sectional, non-interventional, observational, controlled study was conducted in humans. The serum measurement of: vitamin D, parathyroid hormone (PTH), calcium, phosphorus, magnesium and albumin was performed in 49 patients who were consecutively hospitalized for surgery due to fracture(s) (study group), and in 50 patients without fractures, from the various outpatient clinics, and who underwent routine tests (control group).

Results The mean level of 25-hydroxyvitamin $\mathrm{D}(25[\mathrm{OH}] \mathrm{D})$ in patients with fractures was of $23.78 \pm 8.01 \mathrm{ng} / \mathrm{mL}$ ( $61.22 \%$ of patients with fractures had hypovitaminosis $D$ ). The mean $25(\mathrm{OH}) \mathrm{D}$ of the control group was of $37.52 \pm 9.21 \mathrm{ng} / \mathrm{mL}(10 \%$ of the patients had hypovitaminosis D). Most cases of hypovitaminosis (96\%) did not course with secondary hyperparathyroidism (mean PTH $=41.80 \pm 22.75 \mathrm{pg} / \mathrm{mL}$ ).

Conclusion Hypovitaminosis D was found in a significant percentage (61.22\%) of the patients who suffered fractures. Further studies on the subject are needed to better understand the influence of hypovitaminosis $D$ on the occurrence of fractures, as well as the benefits of vitamin $\mathrm{D}$ supplementation in these patients.

\section{Introdução}

O estudo da vitamina D (VIT D) e as consequências de sua deficiência no organismo são temas relativamente novos, e foram conhecidas depois da Revolução Industrial, quando a população rural migrou para a região urbana.

A principal função conhecida da VIT D é manter as concentrações de cálcio e fósforo sérico dentro dos limites normais fisiológicos para a manutenção da maioria das funções metabólicas, a transmissão neuromuscular, e a mineralização óssea. Geralmente, as alterações decorrentes da deficiência da VIT D são subclínicas. ${ }^{1}$

A hipovitaminose $\mathrm{D}$ (HVD) leva à diminuição da mineralização da matriz óssea, o que pode acarretar raquitismo na infância e osteomalácia no adulto, bem como a agravamento da osteoporose. A VIT D tem papel fundamental na via metabólica do cálcio, e sua suplementação está associada ao aumento da massa mineral óssea e à prevenção de fraturas. As fraturas decorrentes da osteoporose resultam em altos custos, com tratamento que se equipara aos das doenças cardiovasculares. ${ }^{2}$

Aproximadamente, um terço da população mundial apresenta níveis de 25 -hidroxivitamina $\mathrm{D}(25(\mathrm{OH}) \mathrm{D})$ menores do que $20 \mathrm{ng} / \mathrm{mL}$, que possivelmente não são os ideais para manter uma boa saúde óssea. ${ }^{3}$ Há evidências de que a HVD seja a deficiência nutricional mais comum do mundo. ${ }^{4}$ Tradicionalmente, considerava-se o Brasil um local em que não havia HVD, por ser um país tropical, ensolarado, e com ampla exposição da população ao sol. Entretanto, estudos recentes $^{5-10}$ vêm demonstrando a HVD nas mais diversas faixas etárias e locais do país. A HVD, que assola a população mundial e inclusive o Brasil, pode ter ocorrido por mudança de hábitos como a proteção contra o sol e o uso de protetores solares, e pela sazonalidade e menor exposição solar em regiões frias, bem como carência alimentar.
Está bem estabelecido na literatura que a HVD predispõe à fragilidade óssea e exerce influência sobre o equilíbrio postural e dinâmico, favorecendo a ocorrência de fraturas. No entanto, até o início deste trabalho, não foram encontrados estudos sobre a dosagem da VIT D na população do sul de Minas, região dos autores deste artigo, e, tampouco, estudos sobre a influência da VIT D na ocorrência das fraturas na população brasileira. Portanto, o objetivo deste estudo foi dosar a VIT D nos pacientes fraturados e avaliar sua relação com as fraturas.

\section{Métodos}

Realizou-se estudo primário, analítico, transversal, não intervencional, observacional, controlado, em seres humanos. Foram selecionados, consecutivamente, 49 pacientes com fraturas (grupo de estudo; 1 paciente do grupo estudo foi excluído por alteração renal) com indicação cirúrgica, e 50 pacientes sem fraturas (grupo de controle), maiores de 18 anos, sem quaisquer restrições quanto à cor ou ao sexo, que concordaram em participar deste estudo. Os pacientes do grupo de estudo foram selecionados de forma consecutiva à sua internação na enfermaria de ortopedia e traumatologia da nossa instituição; e os exames, coletados juntamente com os exames de sangue pré-operatórios de rotina. Os pacientes do grupo de controle foram selecionados dos ambulatórios das diversas especialidades no mesmo hospital, contanto que tivessem alguma indicação ambulatorial de coleta de sangue. Foram excluídos os pacientes: internados por mais de 24 horas; em reposição de VIT D; com doenças endócrinas, genéticas ou metabólicas capazes de alterar o metabolismo normal da VIT D (osteogênese imperfeita, doença de Paget); com insuficiência renal aguda ou crônica; com fraturas patológicas de origem neoplásica ou vigência de tratamento 
neoplásico; em estado de desnutrição; com condições psiquiátricas alimentares (bulimia e anorexia); submetidos a cirurgia bariátrica ou ressecção intestinal; sabidamente com doenças do intestino delgado, doença do trato biliar ou hepático e insuficiência pancreática exócrina, doenças intestinais que interferem na absorção de gordura (espru, amiloidose, etc) e pacientes em uso de medicações com capacidade anti-reabsortiva de gordura: orlistate, celistate e assemelhados.

Todos os pacientes que concordaram em participar espontaneamente do estudo assinaram o termo de consentimento livre e esclarecido. Este estudo foi aprovado pelo Comitê de Ética sob o número CAAE 94612618.6.0000.5102. Os pacientes foram inicialmente submetidos a coleta de dados sociodemográficos, aferição do peso corporal, e medida da estatura em metros, e responderam a um questionário sobre hábitos de proteção solar e tabagismo. A seguir, foram submetidos a punção venosa periférica e coleta de sangue para dosagem de 25(OH)D, paratormônio (PTH), cálcio sérico total e ionizado, fósforo, magnésio, e albumina. A 25(OH)D e o PTH foram dosados pelo método de quimioluminescência. A HVD foi caracterizada de acordo com o posicionamento atual da Sociedade Brasileira de Patologia Clínica e Medicina Laboratorial (SBPC/ML) e da Sociedade Brasileira de Endocrinologia e Metabologia (SBEM). ${ }^{11}$ Assim, os níveis de 25(OH)D acima de $20 \mathrm{ng} / \mathrm{mL}$ foram considerados o valor normal para população saudável (até 60 anos); e, entre $30 \mathrm{ng} / \mathrm{mL}$ e $60 \mathrm{ng} / \mathrm{mL}$, o valor recomendado para grupos de risco como: idosos, gestantes, pacientes osteoporóticos, com histórico de quedas e fraturas, e com causas secundárias de osteoporose (doenças e medicações); e níveis maiores do que $100 \mathrm{ng} / \mathrm{mL}$ foram considerasdos risco de intoxicação.

Os dados foram tabulados no programa Microsoft Excel 2016 (Microsoft Corp., Redmond, WA, EUA) e submetidos a análise estatística. Foram utilizadas medidas de tendência central para variáveis quantitativas e frequências absoluta e relativa para variáveis categóricas. Utilizou-se o programa Minitab (Minitab, LLC, State College, PA, EUA), versão 18.1, e Statistical Package for the Social Sciences (SPSS, IBM Corp., Armonk, NY, EUA), versão 22.0. O nível de significância adotado para os testes estatísticos foi de $5 \%(p<0,05)$.

\section{Resultados}

Os grupos foram semelhantes quanto ao índice de massa corporal (IMC), à idade, e ao sexo (- Tabelas 1 e 2). No grupo de estudo, havia 15 (30,61\%) idosos (maiores de 60 anos),
Tabela 2 Comparação dos grupos com relação ao sexo

\begin{tabular}{|l|l|l|l|l|}
\hline Grupo & Masculino & Feminino & Total & $p$ \\
\hline Estudo & $31(63 \%)$ & $18(37 \%)$ & $49(100 \%)$ & 0,150 \\
\hline Controle & $25(50 \%)$ & $25(50 \%)$ & $50(100 \%)$ & \\
\hline
\end{tabular}

Nota: Teste exato de Fischer.

Tabela 3 Comparação entre o grau de proteção e exposição solar e tabagismo entre os grupos

\begin{tabular}{|l|l|l|l|}
\hline Proteção solar & Controle & Estudo & $p$ \\
\hline Nenhuma & 23 & 23 & \\
\hline Protetor solar e/ou roupa & 27 & 26 & 1 \\
\hline Somente protetor solar & 0 & 0 & \\
\hline Total & 50 & 49 & \\
\hline Tabagismo & & & \\
\hline Sim & 10 & 12 & \\
\hline Não & 40 & 37 & 0,483 \\
\hline Total & 50 & 49 & \\
\hline Exposição solar & & & \\
\hline Diária & 37 & 32 & \\
\hline Eventual & 13 & 19 & 1 \\
\hline Total & 50 & 49 & \\
\hline
\end{tabular}

Nota: Teste $t$.

sendo que 14 (93,33\%) deles apresentaram níveis de VIT D menores do que $30 \mathrm{ng} / \mathrm{mL}$. No grupo de controle, havia 18 (36\%) idosos, e 5 (27,77\%) deles apresentaram níveis de VIT D menores do que $30 \mathrm{ng} / \mathrm{mL}$.

Houve semelhança também quanto à cor da pele dos grupos, predominantemente branca: havia $82 \%$ de brancos no grupo de controle, e $87,75 \%$ no grupo de estudo.

Os grupos se assemelharam ainda quanto ao grau de proteção da pele, e aos hábitos de tabagismo e de exposição ao sol ( - Tabela 3 ).

$\mathrm{O}$ nível médio de PTH do grupo de controle foi de 39 $( \pm 23,70) \mathrm{pg} / \mathrm{mL}$, e do grupo estudo foi de 44,60 ( $\pm 21,80)$ $\mathrm{pg} / \mathrm{mL}(\mathrm{VN}=18,5 \mathrm{pg} / \mathrm{mL}$ a $88 \mathrm{pg} / \mathrm{mL})$. Avaliando-se os dois grupos, observou-se apenas dois casos de hiperparatireoidismo secundário à HVD.

Tabela 1 Distribuição da amostra com relação à idade e ao índice de massa corporal

\begin{tabular}{|c|c|c|c|c|c|c|c|}
\hline & Grupo & $\mathrm{N}$ & Média & Desvio padrão & Mínimo & Máximo & $p$ \\
\hline \multirow[t]{3}{*}{ Idade } & Controle & 50 & 50,40 & 21,3 & 20 & 89 & \\
\hline & & & & & & & 0,983 \\
\hline & Estudo & 49 & 50,30 & 21,5 & 18 & 90 & \\
\hline \multirow[t]{3}{*}{ Índice de massa corporal } & Controle & 50 & 26,13 & 3,69 & 18,39 & 35,1 & \\
\hline & & & & & & & 0,289 \\
\hline & Estudo & 49 & 27,10 & 5,19 & 18,13 & 45,49 & \\
\hline
\end{tabular}

Nota: Teste $t$. 


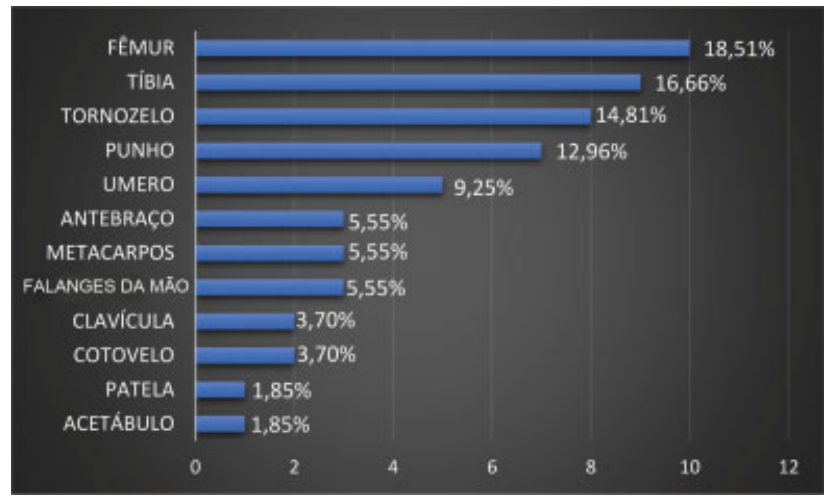

Fig. 1 Distribuição das fraturas (traumas de baixa e alta energia).

Tabela 4 Dosagem da 25-hidroxivitamina D: comparação dos grupos pelo teste $t$

\begin{tabular}{|l|l|l|l|l|}
\hline Grupos & $\mathbf{N}$ & Média $(\mathrm{ng} / \mathrm{mL})$ & Desvio padrão & $\boldsymbol{p}$ \\
\hline Estudo & 49 & 23,78 & 8,01 & $\mathbf{0 , 0 0}^{*}$ \\
\hline Controle & 50 & 37,52 & 9,21 & \\
\hline
\end{tabular}

Nota: *Significância estatística.

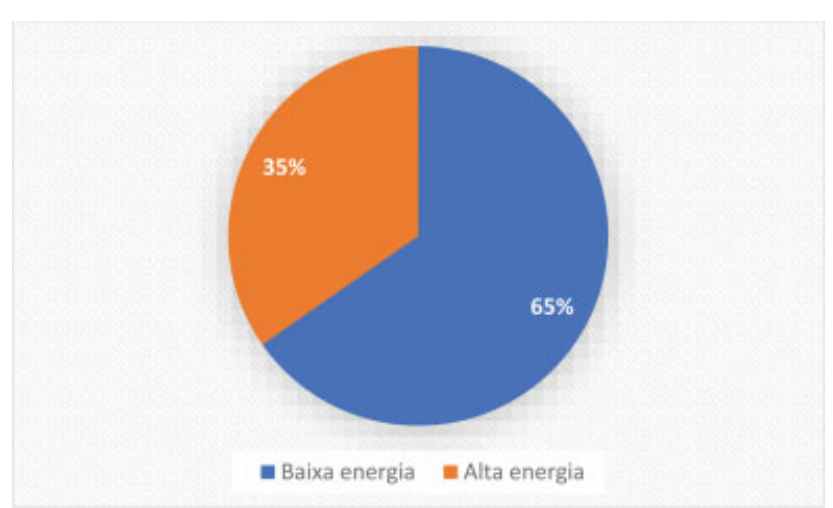

Fig. 2 Distribuição da energia do trauma (porcentagem dos pacientes).

Foram observadas 54 fraturas em 49 pacientes, e 53,68\% delas ocorreram nos membros inferiores (-Figura 1). A média do nível de $25(\mathrm{OH}) \mathrm{D}$ dos grupos pode ser observada na - Tabela 4. Ao todo, $30(61,22 \%)$ pacientes com fraturas (grupo de estudo), e 5 (10\%) pacientes do grupo de controle apresentaram HVD.

A distribuição das fraturas de acordo com a intensidade da energia do trauma está demonstrada na - Figura 2, e sua relação com o grau de suficiência de 25(OH)D, na - Figura 3. A correlação entre a HVD e a ocorrência de fraturas se encontra na tabela 5.

\section{Discussão}

Estudos, incluindo revisão sistemática, ${ }^{12-16}$ avaliaram a dosagem da VIT D nas fraturas, e evidenciaram a importância da HVD na ocorrência delas (prevalência: entre $40 \%$ e $100 \%$ dos casos) . No presente estudo, obervou-se a HVD em 30 (61,22\%) pacientes com fraturas. Houve diferença estatisticamente sig-

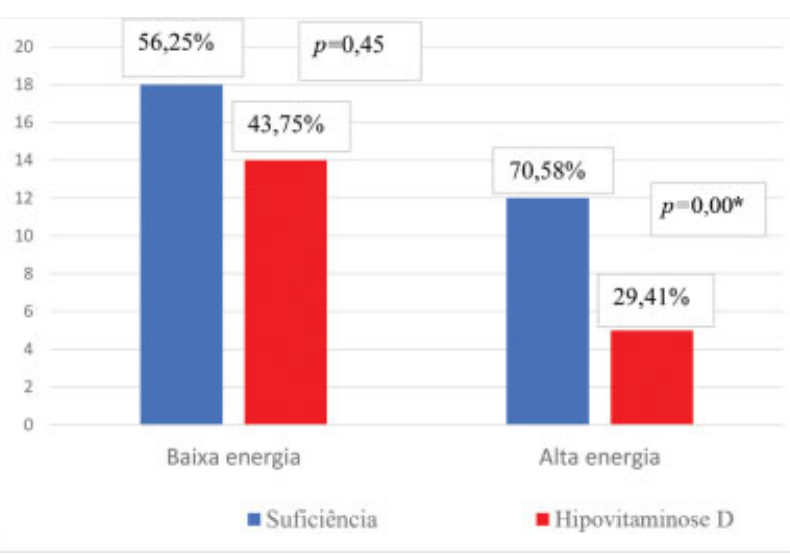

Fig. 3 Distribuição das fraturas de acordo com a intensidade do trauma e grau de suficiência de vitamina D (teste exato de Fischer). Nota: *Significância estatística.

Tabela 5 Correlação entre fraturas e vitamina D pelo coeficiente de Spearman

\begin{tabular}{|l|l|l|}
\hline N (ESTUDO + CONTROLE) & r de Spearman & $p$ \\
\hline 99 & 0,668 & $\mathbf{0 , 0 0}$ \\
\hline
\end{tabular}

Nota: *Significância estatística.

nificativa entre a dosagem de VIT D nos grupos, o que permitiu especular que a HVD foi um agente facilitador das fraturas (-Tabelas 4 e 5). A maioria dos estudos avalia principalmente idosos e mulheres na pós-menopausa, que são populações de risco para osteoporose, ${ }^{7,9}$ e que apresentam um percentual maior de fraturas de baixa energia. ${ }^{12,13,16}$ As fraturas decorrentes de traumas de baixa energia costumam ser espontâneas, por quedas da própria altura ou por mecanismos torcionais, e ocorrem principalmente no fêmur proximal, no rádio distal, e na coluna ${ }^{12,16,17}$ especialmente no idoso, em que é frequente o episódio dentro do domicílio. Comumente, são lesões únicas, e as que mais requerem tratamento cirúrgico. ${ }^{13}$ Nos estudos que avaliaram exclusivamente as fraturas de baixa energia, encontrou-se maior frequência de fraturas de fêmur proximal ${ }^{12,16}$ ou punho. ${ }^{15}$ Este estudo avaliou todas as fraturas que chegaram consecutivamente no pronto-socorro, ou seja, as de baixa e alta energia, que foram incluídas contanto que tivessem indicação cirúrgica. Por um lado, isto poderia ser um fator limitante deste estudo, uma vez que as fraturas sem indicação cirúrgica não foram catalogadas; porém, evitou-se, assim, punção venosa que fosse para fim exclusivo deste estudo. As fraturas de baixa energia também foram predominantes neste trabalho ( - Figura 2), mas, diferentemente de outras publicações ${ }^{12,15,16}$, a maior incidência de fraturas de baixa energia ocorreu em tornozelos (8 casos; $14,81 \%$ das fraturas). Esta discrepância talvez se justifique pelo fato de que, neste trabalho, diferentemente de outros $^{12,13,15,16}$, a população masculina foi maioria $(63 \%$ dos casos), e também porque, neste estudo, observou-se média de idade (de 50,3 anos) muito inferior ao encontrado em outros trabalhos ${ }^{12,13,15,16}$. Portanto, a amostra dste estudo foi composta por uma maioria de pacientes do sexo e de uma faixa etária em que são menos frequentes as fraturas proximais do fêmur. 
As fraturas de alta energia geralmente são decorrentes de acidentes ocorridos fora de casa, como os automobilísticos; são menos avaliadas, mas esses pacientes também costumam ter índices de HVD muito elevados. ${ }^{14}$ Nas fraturas ocorridas por trauma de alta energia neste estudo, houve 29,41\% de HVD (-Figura 3).

Embora seja desconhecido o efeito da VIT D na fratura já ocorrida e não haja evidências concretas de que ela acelere o processo de consolidação, é inegável seu efeito no aumento da vascularização do calo ósseo, ${ }^{18}$ da resistência óssea e da densidade mineral, além de no aumento do volume de calo ósseo. ${ }^{10,14}$ Além disto, um estudo ${ }^{19}$ relatou HVD em $83 \%$ de casos de pseudoartrose e retardo de consolidação. Em um estudo retrospectivo, Gorter et al. ${ }^{15}$ avaliaram 617 pacientes com fraturas ( $40 \%$ de prevalência de HVD), e observaram que a consolidação sofreu influência dos níveis de VIT D no momento da ocorrência.

É habitual que, diante de fraturas, os níveis de VIT D caiam agudamente pelo seu consumo no foco da fratura, especialmente se o nível inicial já é baixo. Esta queda ocorre entre uma e três semanas após a fratura. ${ }^{14}$ Os níveis iniciais de VIT D caem em torno de $20 \%$ ao final da primeira semana. ${ }^{20}$ Considera-se este detalhe um fator de suma importância nos estudos de prevalência de HVD nas fraturas, mas parte significativa dos trabalhos não menciona o momento exato em que foram avaliados os níveis de 25(OH)D. Neste estudo, essa avaliação ocorreu em até 24 horas da fratura. Este detalhe também tem importância para a suplementação mais rigorosa do paciente fraturado e com HVD .

As quedas representam um marcador de morbidade e mortalidade, especialmente nos idosos, e ocasionam fraturas em cerca de $10 \%$ das vezes. ${ }^{21}$ No Brasil, pela osteoporose, acomete principalmente mulheres de regiões metropolitanas. ${ }^{22}$ Há elevada prevalência da HVD, em especial nos idosos. Observou-se neste estudo que 93,33\% dos idosos com fraturas apresentaram HVD. As fraturas decorrentes de quedas, principalmente do quadril, levam à morte em cerca de $20 \%$ dos casos, e, em $50 \%$ dos casos não fatais, levam o idoso a se tornar dependente. ${ }^{23} 0$ tratamento da HVD nos idosos reduz as quedas em $20 \%$, e, a cada 15 pacientes tratados, previne-se 1 queda. ${ }^{24}$ Níveis adequados de VIT D diminuem o risco de fraturas em $20 \%{ }^{25}$ Observou-se também que o uso associado de VIT D e cálcio diminuiu em $15 \%$ a chance de desenvolver fraturas em geral, e em $30 \%$, as fraturas do quadril. ${ }^{26}$

O cirurgião ortopédico deve estar preparado para reconhecer a HVD e saber rastreá-la e tratá-la, ou encaminhar o paciente. ${ }^{27} \mathrm{Na}$ prática diária, os pacientes internados com fraturas para se submeter ao tratamento cirúrgico realizam exames pré-operatórios que habitualmente não envolvem a dosagem da VIT D. Dada a importância da VIT D na força muscular e no equilíbrio, e seu papel (ainda que questionável) na consolidação das fraturas, sugere-se que, quando possível, a dosagem de VIT D (exame relativamente caro e não coberto pelo SUS) faça parte da rotina pré-operatória, em especial nos idosos ou mulheres na pós-menopausa com fraturas, bem como seja feita a sua reposição, caso necessário. A HV D está presente em todas as faixas etárias e grupos populacionais, em especial entre os idosos brasileiros, que dependem da síntese cutânea para obter suficiência. ${ }^{5,6}$ Embora a exposição ao sol possa aumentar os níveis de VIT D, esta gestão orgânica para a suficiência de VIT D tem-se mostrado desfavorável diante dos efeitos carcinogênicos potenciais dos raios ultravioleta e das mudanças nos hábitos (proteção solar), e também porque as estações do ano e a latitude geográfica podem impedir a exposição apropriada aos raios solares. Portanto, a suplementação dietética tem sido o método preferido para gerenciar os níveis de VIT D. Um estudo ${ }^{24}$ realizado com jovens saudáveis demonstrou que o uso de VIT D é seguro e livre de efeitos adversos graves, até mesmo em doses dez vezes maiores do que a recomendado. A política de suplementação de alimentos, como leite, suco de laranja e manteiga, com VIT D demonstrou ser eficiente para elevar as concentrações plasmáticas de $25(\mathrm{OH}) \mathrm{D}$ nos países em que é praticada. ${ }^{6}$ Políticas semelhantes poderiam ser utilizadas também em nosso país, com benefícios potenciais para nossa população geriátrica. Dado o baixo custo, a segurança e o benefício demonstrado de maiores concentrações de 25 $(\mathrm{OH}) \mathrm{D}$, a suplementação deveria ser uma prioridade de saúde pública. ${ }^{28}$ Apesar de se considerar barata a suplementação de VIT D nos alimentos, no Brasil, ela é feita em alimentos específicos que não fazem parte da alimentação rotineira do idoso. Até que a suplementação de VIT D de forma mais ampla se torne realidade no Brasil, o traumatologista tem o dever de conhecer e corrigir a HVD, em especial nos idosos atendidos com fraturas. São raros os eventos de intoxicação pela VIT D. Deve-se, então, ponderar que, em casos selecionados, a suplementação, ainda que de certa forma indiscriminada (nem sempre é possível dosar a VIT D), pode trazer benefícios que superariam os riscos da sua deficiência e os elevados custos associados às fraturas.

\section{Conclusão}

A HVD foi encontrada em um percentual expressivo (61,22\%) dos pacientes que sofreram fraturas. Mais estudos sobre o assunto são necessários para se compreender melhor a influência da hipovitaminose $\mathrm{D}$ na ocorrência das fraturas e os benefícios da suplementação com VIT D nesses pacientes.

\section{Suporte Financeiro}

Não houve suporte financeiro de fontes públicas, comerciais, ou sem fins lucrativos.

\section{Conflito de Interesses}

Os autores declaram não haver conflito de interesses.

\section{Referências}

1 Maeda SS, Fortes EM, Oliveira UM, Borba VC, Lazaretti-Castro M. Hypoparathyroidism and pseudohypoparathyroidism. Arq Bras Endocrinol Metabol 2006;50(04):664-673

2 Aziziyeh R, Amin M, Habib M, et al. The burden of osteoporosis in four Latin American countries: Brazil, Mexico, Colombia, and Argentina. J Med Econ 2019;22(07):638-644 
3 Federação Brasileira das Associações de Ginecologia e Obstetrícia (FEBRASGO) A importância da vitamina D na saúde da mulher. São Paulo: FEBRASGO; 2018. (Série Orientações e Recomendações FEBRASGO, n. ${ }^{\circ} 14$ )

4 Hossein-nezhad A, Holick MF. Vitamin D for health: a global perspective. Mayo Clin Proc 2013;88(07):720-755

5 Bandeira F, Griz L, Freese E, et al. Vitamin D deficiency and its relationship with bone mineral density among postmenopausal women living in the tropics. Arq Bras Endocrinol Metabol 2010;54 (02):227-232

6 Saraiva GL, Cendoroglo MS, Ramos LR, et al. Prevalência da deficiência, insuficiência de vitamina D e hiperparatiroidismo secundário em idosos institucionalizados e moradores na comunidade da cidade de São Paulo, Brasil. Arq Bras Endocrinol Metabol 2007;51(03):437-442

7 Peters BS, dos Santos LC, Fisberg M, Wood RJ, Martini LA. Prevalence of vitamin D insufficiency in Brazilian adolescents. Ann Nutr Metab 2009;54(01):15-21

8 Paiva CAC, Bettinelli LA, Portela MR, Pasquallotti A. Prevalência de hipovitaminose D e sua severidade em idosos institucionalizados no município de passo fundo. Rev Cont Saúde 2011;10(20): $1243-1250$

9 Santos EP Junior, Fernandes DC, Almeida AT, Borges FA, Novaes JA. Epidemiologia da vitamina D. Reva Cient ITPAC, Araguaína. 2011; 4(03). Disponível em: https://assets.itpac.br/arquivos/Revista/43/ 2.pdf

10 Maeda SS, Borba VVC, Camargo MB, et al. Recomendações da Sociedade Brasileira de Endocrinologia e Metabologia (SBEM) para o diagnóstico e tratamento da hipovitaminose D. Arq Bras Endocrinol Metabol 2014;58(05):411-433

11 Sociedade Brasileira de Patologia Clínica e Medicina Laboratorial (SBPC/ML) e Sociedade Brasileira de Endocrinologia e Metabologia (SBEM) Posicionamento de Intervalos de Referência da Vitamina D - 25(OH)D. [acesso em 03/02/2019]. Disponível em: http://www.sbpc.org.br/noticias-e-comunicacao/novos-intervalos-de-referencia-de-vitamina-d/

12 Simonelli C, Weiss TW, Morancey J, Swanson L, Chen YT. Prevalence of vitamin $D$ inadequacy in a minimal trauma fracture population. Curr Med Res Opin 2005;21(07):1069-1074

13 Mow TC, Stokes CM, Sutherland AG. Patients presenting with fractures are likely to be vitamin D deficient: are we getting enough sun? ANZ J Surg 2015;85(10):766-769

14 Sprague S, Petrisor B, Scott T, et al. What is the role of vitamin d supplementation in acute fracture patients? A systematic review and meta-analysis of the prevalence of hypovitaminosis D and supplementation efficacy. J Orthop Trauma 2016;30(02):53-63
15 Gorter EA, Krijnen P, Schipper IB. Vitamin D status and adult fracture healing. J Clin Orthop Trauma 2017;8(01):34-37

16 Devigili N Jr, Botega L, Back SRS, Stipp WN, Back Netto M. Prevalência da deficiência de vitamina $\mathrm{D}$ em pacientes com fraturas ocasionadas por trauma de baixa energia. Rev Bras Ortop 2019;54(01):69-72

17 Milzman DP, Boulanger BR, Rodriguez A, Soderstrom CA, Mitchell KA, Magnant CM. Pre-existing disease in trauma patients: a predictor of fate independent of age and injury severity score. J Trauma 1992;32(02):236-243, discussion 243-244

18 Omeroğlu S, Erdoğan D, Omeroğlu H. Effects of single high-dose vitamin D3 on fracture healing. An ultrastructural study in healthy guinea pigs. Arch Orthop Trauma Surg 1997;116(1-2):37-40

19 Brinker MR, O'Connor DP, Monla YT, Earthman TP. Metabolic and endocrine abnormalities in patients with nonunions. J Orthop Trauma 2007;21(08):557-570

20 Ettehad H, Mirbolook A, Mohammadi F, Mousavi M, Ebrahimi H, Shirangi A. Changes in the serum level of vitamin d during healing of tibial and femoral shaft fractures. Trauma Mon 2014;19(01): e10946

21 Gallagher JC. The effects of calcitriol on falls and fractures and physical performance tests. J Steroid Biochem Mol Biol 2004;8990(1-5):497-501

22 Pinheiro MM, Ciconelli RM, Jacques NO, Genaro PS, Martini LA, Ferraz MB. O impacto da osteoporose no Brasil: dados regionais das fraturas em homens e mulheres adultos - The Brazilian Osteoporosis Study (BRAZOS). Rev Bras Reumatol 2010;50(02):113-120

23 Mello RG, Schneider RH, Collares FM, Dalacorte RR. Vitamina D e prevenção de quedas em idosos: uma revisão sistemática. Sci Med (Porto Alegre) 2010;20(02):200-206

24 Bischoff-Ferrari HA, Dietrich T, Orav EJ, Dawson-Hughes B. Positive association between 25-hydroxy vitamin $D$ levels and bone mineral density: a population-based study of younger and older adults. Am J Med 2004;116(09):634-639

25 Zittermann A, Gummert JF. Nonclassical vitamin D action. Nutrients 2010;2(04):408-425

26 Weaver CM, Alexander DD, Boushey CJ, et al. Calcium plus vitamin D supplementation and risk of fractures: an updated metaanalysis from the National Osteoporosis Foundation. Osteoporos Int 2016;27(01):367-376

27 Nino S, Soin SP, Avilucea FR. Vitamin D and Metabolic Supplementation in Orthopedic Trauma. Orthop Clin North Am 2019;50 (02):171-179

28 Bischoff-Ferrari HA, Conzelmann M, Stähelin HB, et al. Is fall prevention by vitamin $\mathrm{D}$ mediated by a change in postural or dynamic balance? Osteoporos Int 2006;17(05):656-663 